\title{
Review Article \\ Probiotic Species in the Modulation of Gut Microbiota: An Overview
}

\author{
Md. Abul Kalam Azad $\mathbb{D},{ }^{1,2}$ Manobendro Sarker, ${ }^{3}$ Tiejun Li $\mathbb{D},{ }^{1}$ and Jie Yin $\mathbb{D}^{1,2}$ \\ ${ }^{1}$ Key Laboratory of Agro-Ecological Processes in Subtropical Region, Institute of Subtropical Agriculture, \\ Chinese Academy of Sciences, National Engineering Laboratory for Pollution Control and Waste Utilization in \\ Livestock and Poultry Production, Changsha, Hunan 410125, China \\ ${ }^{2}$ University of Chinese Academy of Sciences, Beijing 100049, China \\ ${ }^{3}$ Department of Food Engineering and Technology, State University of Bangladesh, Dhaka 1205, Bangladesh
}

Correspondence should be addressed to Jie Yin; yinjie2014@126.com

Received 25 February 2018; Accepted 29 March 2018; Published 8 May 2018

Academic Editor: Lidong Zhai

Copyright (C) $2018 \mathrm{Md}$. Abul Kalam Azad et al. This is an open access article distributed under the Creative Commons Attribution License, which permits unrestricted use, distribution, and reproduction in any medium, provided the original work is properly cited.

\begin{abstract}
Probiotics are microbial strains that are beneficial to health, and their potential has recently led to a significant increase in research interest in their use to modulate the gut microbiota. The animal gut is a complex ecosystem of host cells, microbiota, and available nutrients, and the microbiota prevents several degenerative diseases in humans and animals via immunomodulation. The gut microbiota and its influence on human nutrition, metabolism, physiology, and immunity are addressed, and several probiotic species and strains are discussed to improve the understanding of modulation of gut microbiota. This paper provides a broad review of several Lactobacillus spp., Bifidobacterium spp., and other coliform bacteria as the most promising probiotic species and their role in the prevention of degenerative diseases, such as obesity, diabetes, cancer, cardiovascular diseases, malignancy, liver disease, and inflammatory bowel disease. This review also discusses a recent study of Saccharomyces spp. in which inflammation was prevented by promotion of proinflammatory immune function via the production of short-chain fatty acids. A summary of gut microbiota alteration with future perspectives is also provided.
\end{abstract}

\section{Introduction}

Alteration of the gut microbiota with probiotic species is very prominent in human and animal disease treatment. The potential of probiotic species has recently motivated researchers to examine the production of probiotic foods and the modulation of the gut microbiota. The importance of consumption of probiotic foods with a specific mix of bacteria has been widely studied since the beginning of the 20th century, and yogurt has drawn attention to maintaining good health via development of the digestive system and the prevention of various degenerative diseases [1-3].

The word "probiotic" comes from Greek and means "for life." In 1954, Ferdinand Vergin conceived the term "probiotic" in an article entitled "Anti-und Probiotika," in which several microorganisms were studied to make a list of useful bacteria and to determine the detrimental effects of antibacterial agents and antibiotics on the intestinal microbiota [4].
A few years later, Lilly and Stillwell described probiotics as beneficial microorganisms that exert growth-promoting factors for other microorganisms [5]. The term "probiotics" has been modified over time and with research into their application and clinical trials in various human and animal models. According to the Food and Agriculture Organization (FAO) and the World Health Organization (WHO), probiotics are live strains of microorganisms that confer health benefits upon the host when administrated in adequate amounts [6], and this definition is followed by the International Scientific Association for Probiotics and Prebiotics (ISAPP) $[7,8]$. However, researchers continue to develop new probiotic species, even though probiotic species have long been used for human health improvement. Most probiotic products today are developed with Bifidobacteria, Lactobacilli, and other lactic acid bacteria, such as Lactococci and Streptococci. Other promising probiotic strains include the bacterial genera Bacillus, Escherichia, and Propionibacterium 
and some other yeast genera, mainly Saccharomyces. Probiotics are usually considered to be safe for human health with limited adverse effects [9]. Several species and strains of Lactobacilli, including Lactobacillus acidophilus, Lactobacillus casei, Lactobacillus rhamnosus, and Lactobacillus helveticus, have been extensively studied in the prevention of human and animal diseases. These probiotic species are able to change the population of microorganisms in the gut microbiota and control the functioning of the ecosystem of gut microbiota. In earlier studies, considerable evidence of clinical trials of probiotics in animal and human models has reported suitability for the treatment of a variety of diseases, and this number continues to grow.

The human gut is a complex ecosystem in which nutrients, the microbiota, and host cells interact extensively. The relationships between these microorganisms and host cells were long considered only from a pathogenic point of view because toxins invade the gut mucosa and translocate, disseminate, and cause systemic infections [10]. However, no attention was paid to the majority of gut microorganisms and their relationship with host health. Several studies have reported beneficial interactions between the commensal microbiota and the human body and have indicated that the microbiota acts as a real partner. A deeper understanding of the gut microbiota and its role is necessary for future healthcare strategies. In this regard, extensive study of the potential use of selected probiotic bacteria species and their strains is desperately needed for the prevention and treatment of numerous human and animal diseases [11-14].

The relationship between health and the composition of the gut microbiota has raised interest in the modulation of the gut microbiota by administration of probiotic species for the prevention of some diseases in humans and animals. This review focuses on the gut microbiota and several probiotic species that have been extensively studied in the modulation of the gut microbiota and prevention of degenerative diseases.

\section{Gut Microbiota}

The term "gut microbiota" was first introduced to the scientific community by Joshua Lederberg who called it "the ecological community of commensal, symbiotic, and pathogenic microorganism that literally share our body space and have been all but ignored as determinants of health diseases" [31]. The human body consists of trillions of microbes, mostly within the gastrointestinal tract (i.e., the small intestine and colon). Using a $70 \mathrm{~kg}$ man as a reference, $3.8 \times 10^{13}$ microbes are reported to have a total weight of $0.2 \mathrm{~kg}$ [32]. The gut microbiota can ferment nondigestible carbohydrates, which are well known as prebiotics, including fructooligosaccharide, oligofructose, inulin, galactose, and xylose, that contain oligosaccharides to fulfill energy requirements. The microbes in the host body have a significant influence on the metabolism, physiology, and immune development and function, whereas symbiotic functions include the synthesis of vitamins, protection from pathogenic colonization as a regulatory immune system via modulation of gastrointestinal hormone release function, and regulation of brain behavior in terms of neuronal signaling [33-38]. The improvement of culture-independent and molecular high-throughput techniques favor the identification of previously unknown bacteria, which would provide novel insights into the functional capacity and compositional diversity of some of the fecal microbiota. In addition, several studies have suggested that disorders such as colorectal cancer, inflammatory bowel disease (IBD), alcoholic and nonalcoholic fatty liver diseases, obesity, type 2 diabetes, oxidative stress-related disease, and immune-mediated diseases are associated with disease-specific dibiotic of altered microbiota compositions [15, 39-43]. Modification of the gut microbiota has thus gained more attention as a potential treatment for several diseases in humans and animals.

\section{Modulation of Gut Microbiota and Probiotic Species}

The gut microbiota includes bacteria, fungi, archaea, protozoa, and viruses that interact with the host and each other to affect the host's physiology and health [44]. The gut bacteria play significant roles in human health, including vitamin B synthesis, improvement in digestion, and promotion of angiogenesis and nerve function [45]. In addition, modification of the gut microbiota can be harmful when the gut ecosystem undergoes severe abnormal changes. The bacterial species found in the human gut microbiome include mostly three phyla: Bacteroidetes (Porphyromonas, Prevotella), Firmicutes (Ruminococcus, Clostridium, and Eubacteria), and Actinobacteria (Bifidobacterium). Lactobacilli, Streptococci, and Escherichia coli are found in small numbers in the gut. However, alteration of the gut microbiota composition can lead to multiple diseases in humans and animals [21, 22, 28, 30].

Current evidence supports a link between the activity and composition of the gut microbiota and human health and disease. Furthermore, the gut microbiota composition is likely to affect many organ systems, including the cardiovascular, neural, immune, and metabolic systems. The gut microbiota composition is altered in many disease states, such as cardiovascular disease, cancer, malignancy, type 2 diabetes mellitus, obesity, colitis, asthma, psychiatric disorders, inflammatory disorders, disorders of the gut-brain axis, and numerous immune disorders [15, 40, 41, 46-48]. Modulation of the gut microbiota facilitates a number of health problems; probiotic feeding with a high-fat diet showed alteration of the gut microbiota composition with a decrease in the grampositive bacteria phyla Firmicutes and Actinobacteria in mice [49]. In contrast, in a mouse model of hyperlipidemia, the probiotic administration of Lactobacillus led to significant changes in the microbiota composition, including an increased abundance of Bacteroidetes and Verrucomicrobia and a reduced ratio of Firmicutes [50]. It is evident that probiotic species play important roles in maintaining the gut microbiota ecosystem in humans and animals.

\section{Bacteria Species}

Table 1 presents the most significant results of various studies on the influence of probiotic bacteria species or strains on 
TABLE 1: Some bacterial strains used in gut microbiota modulation.

\begin{tabular}{|c|c|c|c|c|}
\hline Bacteria strains & Disease model & Disease & Outcomes & References \\
\hline L. acidophilus & Eight-week-old male C57BL/mice & IBD & $\begin{array}{c}\uparrow \text { IL-10, Treg } \\
\downarrow \text { IL-6, IL-1 } \beta, \text { IL-17 }\end{array}$ & [15] \\
\hline L. acidophilus (NCK2025) & Generation of TS4Cre $\times \mathrm{APC}^{\operatorname{lox} 468}$ mice & CRC & $\begin{array}{c}\uparrow \mathrm{IL}-10, \mathrm{IL}-12 \\
\downarrow \text { Treg }\end{array}$ & [16] \\
\hline L. acidophilus & Female BALB/c mice & Crohn's disease & $\begin{array}{c}\uparrow \text { IL-17 } \\
\downarrow \text { Th17 function, IL-23 }\end{array}$ & [17] \\
\hline L. acidophilus & $\mathrm{BALB} / \mathrm{c}$ mice & Ulcerative colitis & $\begin{array}{c}\uparrow \text { Lactobacilli, } \\
\text { Bifidobacteria } \\
\downarrow \text { S. aureus }\end{array}$ & [18] \\
\hline L. casei BL23 & Female C57BL/6 mice & CRC & $\begin{array}{c}\uparrow \text { Th17, Th } 22 \text {, IL-10, and } \\
\text { IL-22 } \\
\downarrow \text { Treg }\end{array}$ & {$[19,20]$} \\
\hline L. fermentum FTDC 812 & Eight week old $\mathrm{BALB} / \mathrm{c}$ mice & Hypercholesterolemia & $\uparrow$ Lactobacillus & [21] \\
\hline L. johnsonii & Male C57BL/6 mice & Acute live injury & $\uparrow$ IL-22, Lactobacillus & {$[22]$} \\
\hline L. plantarum CCFM10, RS15-3 & 58 week $\mathrm{BALB} / \mathrm{c}$ mice & Oxidative stress & $\uparrow$ Bacteroidetes, Firmicutes & [23] \\
\hline $\begin{array}{l}\text { L. acidophilus } \\
\text { B. cereus, B. infantis }\end{array}$ & Eight week SPE male SD mice & $\begin{array}{l}\text { Nonalcoholic fatty } \\
\text { liver disease }\end{array}$ & $\begin{array}{c}\uparrow \text { E. coli, Enterococcus, } \\
\downarrow \text { Bifidobacteria, } \\
\text { Bacteroides, and } \\
\text { Lactobacillus }\end{array}$ & {$[24]$} \\
\hline $\begin{array}{l}\text { L. acidophilus } \\
\text { L. rhamnosus, B. bifidum }\end{array}$ & Eight week C57BL/6 mice & Type 2 diabetes & $\begin{array}{c}\uparrow \text { Firmicutes, } \\
\text { Actinobacteria } \\
\downarrow \text { Bacteroidetes }\end{array}$ & [25] \\
\hline B. breve IPLA20004 & Human colon & Inflammatory & $\uparrow$ IL-8, IL-10, IL-12 & {$[26]$} \\
\hline E. coli Nissle 1917 & Male C57BL/6J mice & $\begin{array}{l}\text { Chronic } \\
\text { inflammation }\end{array}$ & $\begin{array}{c}\uparrow \text { IL-10, tight-junction } \\
\downarrow \text { IL-17 }\end{array}$ & [27] \\
\hline S. boulardii & Adult $\mathrm{BALB} / \mathrm{c}$ mice & Acute liver failure & $\begin{array}{c}\uparrow \text { Bacteroidetes } \\
\downarrow \text { Firmicutes, Proteobacteria }\end{array}$ & {$[28]$} \\
\hline S. boulardii & Six week C57BL/6 mice & Type 2 diabetes & $\begin{array}{l}\uparrow \text { Firmicutes, } \\
\text { Proteobacteria, and } \\
\text { Fibrobacteria }\end{array}$ & [29] \\
\hline E. hirae & C57BL/6J mice & Cancer & $\uparrow$ Th 17 cell response & [30] \\
\hline
\end{tabular}

modulation of the gut microbiota in various models and diseases.

4.1. Lactobacillus. Most studies of probiotic species in biomedical research have examined the lactic acid bacteria group. In gut microbiota studies, Lactobacillus has been reported as the most prominent probiotic from the lactic acid bacteria group. Changes in the composition, diversity, and function of the gut microbiota by probiotic species have been studied using tools and techniques including targeted, culture-dependent methods and metagenomics sequencing. However, a few studies have demonstrated the associations of probiotic species with altered gut microbiota composition. A recent metagenomic analysis of 8-week-old Swiss mice fed a high-fat diet showed that treatment with a probiotic mixture of Lactobacillus and Bifidobacterium (L. rhamnosus, L. acidophilus, and Bifidobacterium bifidum) significantly altered the composition of the gut microbiota and increased insulin sensitivity. Several authors have reported that mice with a high-fat diet with probiotic species had a lower population of Firmicutes, Actinobacteria, and Bacteroides than untreated mice [25]. Similar work on obese mice revealed that several Lactobacillus spp., Bifidobacterium spp., and other coliform bacteria increased the gut microbiota composition in mice with a high-fat diet treated with various Lactobacillus probiotic strains (L. acidophilus IMV B-7279, L. casei IMV B7280, $B$. animalis VKL, and $B$. animalis VKB). In addition, the gut microbiota composition of obese mice treated with $L$. casei, L. delbrueckii subsp. bulgaricus, and B. animalis showed a significant decrease in microscopic fungi [51]. Probiotic species of Lactobacillus may improve gastrointestinal barrier function by the proliferation of some harmful bacteria [24, 52]. Intestinal permeability can be achieved with an increase in the intestinal tight-junction protein occludin. After a change in the gut microbiota composition with a probiotic, mice with a high-fat diet were reported to show an increase in the expression of the tight-junction protein, proglucagon mRNA, and reduced intestinal expression of the pattern recognition receptors $\mathrm{CD}-14$ and NOD1. It also leads to a reduction in the circulating level of lipopolysaccharide and an increase in glucagon-like peptide 1. In addition, probiotic-treated mice have shown increases in lipoproteinlipase-dependent triglyceride storage in adipose tissue and adipocyte triacylglycerol accumulation [25, 53]. Probiotic Lactobacillus strains have been found to increase gastrointestinal barrier function by the proliferation of harmful 
bacteria in nonalcoholic fatty acid liver diseases and IBD $[15,24]$.

An accumulating body of research on probiotics provides evidence that $\mathrm{T}$ regulatory (Treg) cells play a crucial role in maintaining immune homeostasis in many diseases. Treg cells secrete IL-10, IL-17, and IL-22 (anti-inflammatory cytokine) which are important for maintenance of homeostasis [54-56]. Commensal Lactobacillus species can restore homeostasis in intestinal disorders and thus play a protective role against inflammatory diseases. A recent study showed that a probiotic species of Lactobacillus acidophilus (L. acidophilus) administered for modulation of dextran sulfate sodium-induced colitis restored the balance of inflammatory cytokines and Th17/Treg cells [15]. The authors also reported that $L$. acidophilus suppressed proinflammatory cytokines such as IL-6, tumor necrosis factor-a, and IL-1b in colon tissues. In addition, in vitro treatment by L. acidophilus directly induced the production of IL-10 and Treg cells and suppressed the production of IL-17. Similarly, a probiotic strain of L. acidophilus isolated from a normal human intestinal tract and orally administrated in mice with dextran sulfate sodium-induced colitis suppressed the colitis-associated response of the IL-23/Th17 axis and reduced the secretion of proinflammatory cytokines [17]. Furthermore, based on Treg cell modulation and Th17-biased immune response in regulatory cytokines, the probiotic strain of Lactobacillus spp. showed beneficial effects in preventing cancer and intestinal inflammation $[16,19,57,58]$. Similarly, a probiotic strain of $L$. plantarum TN8 reduced the proinflammatory cytokine expression and also regulated the intestinal immune system of Wistar rats with trinitrobenzene sulfuric acidinduced colitis [59]. The same probiotic strains (L. plantarum TN8) also showed anti-inflammatory properties by inducing production of IL-10 and a small amount of IL-12 cytokines [60].

4.2. Bifidobacterium. Bifidobacterium is important in gut microbiota studies and has long been used as a probiotic to alleviate various diseases by changing the gut microbiota composition. Like other Lactobacillus, Bifidobacterium can also inhibit harmful bacteria, improve gastrointestinal barrier function, and suppress proinflammatory cytokines [24]. Recent studies have demonstrated that Bifidobacterium alters the function of dendritic cells to regulate the intestinal immune homeostasis to harmless antigens and bacteria or initiate protective measures against pathogens. It also has the potential to control various intestinal diseases, like IBD, cancer, and allergies [61-63]. The probiotic Bifidobacterium has shown metabolic capacity in gut bacteria and can increase the proportion of beneficial bacteria in the gut microbiota by cross-feeding. According to Turroni et al. [64], Bifidobacterium bifidum significantly increased metabolic activity when cocultured with Bifidobacterium breve. This coculture of probiotic bacteria affected the metabolic shift in the gut microbiota by increasing the production of shortchain fatty acids rather than by changing the gut microbiota composition. Colonic mucus is a physical barrier that consists of gut microbiota and is maintained by an extensively glycosylated mucin-2 network. In vivo, the ability of bacteria-sized beads to penetrate the mucus layer was greater in mice fed a Western-style diet than in chow-fed mice, which indicated slower mucus growth in the mice fed a Western-style diet due to host metabolic factors. It is worth noting that the abundance of Firmicutes increased and that of Bacteroidetes and Actinobacteria were reduced in the colonic lumen of mice fed a Western-style diet. A study with probiotic treatment showed that Bifidobacterium longum NCC 2705 (B. longum) prevented mucus production [65]. Moreover, Bifidobacterium exerts a positive effect via hormonal signaling in the gut-brain microbiome axis to improve memory function, including brain-derived neurotropic factor and N-methyl-D-aspartate receptor expression. It has been reported that a combination of Lactobacilli and Bifidobacterium decreased acute stress and depression $[66,67]$. However, the understanding of the molecular mechanism is beyond the scope of this study.

4.3. Other Bacteria Species. Like other probiotic species, Escherichia coli, a gram-negative bacterium in the Enterobacteriaceae family, is a well-known probiotic strain with some beneficial effects on gut microbiota homeostasis. The nonpathogenic strain Escherichia coli Nissle $(\mathrm{EcN})$ is one of the most used probiotic strains in gut microbiota homeostasis. It has been shown that $\mathrm{EcN}$ can stimulate the production of human $\beta$-defensin 2 , which can protect the mucosal barrier against adhesion and invasion by pathogenic commensals $[68,69]$. In addition, several in vivo and in vitro studies have shown that $\mathrm{EcN}$ has a protective function against Salmonella, Shigella, Candida, and some other invasive commensals and may restore damaged epithelium by modulation of tightjunction and zonula occludens proteins [70]. However, outer membrane vesicles (OMVs) released by gram-negative bacteria play a vital role in the signaling process of the intestinal gut mucosa. The release of OMVs begins a mechanism to deliver some active compounds and microbial proteins to the host body without intercellular contact. It was recently demonstrated that OMVs trigger the host immune and defense responses of the probiotic strain $\mathrm{EcN}$, which entered intestinal cells via clathrin-mediated endocytosis. In fact, in vitro and ex vivo studies have demonstrated expression of antimicrobial peptides and modulation of the cytokine/chemokine response of gut epithelial and gut immune cells when the probiotic strain EcN induced OMVs. Moreover, these OMVs promote the upregulation of the tight-junction proteins of zonula occludens and claudin-14, but down-regulation of claudin-2 reduces gut permeability and supports intestinal barrier functions in intestinal epithelial cell lines [71, 72]. Finally, the probiotic strain $\mathrm{EcN}$ is also involved in the intestinal microbiota immune response, including macrophages, epithelial cells, dendritic cells, and upregulation of proinflammatory cytokines (IL-6, IL-8, and IL-1 $\beta$ ) [71].

Enterococcus are gram-positive bacteria in the lactic acid bacteria family. Some strains of Enterococcus exert antibioticinduced dysbiosis and act as antitumor or anticancer agents and modulate the immune system. It has been found that culture of $E$. faecium strain from human intestinal epithelium increased the bactericidal effects against enteroaggregative $E$. coli, membrane damage, and cell lysis [73, 74]. Fusco et al. [74] characterized intestinal cytokine expression in epithelial 
cells and reported that intestinal cytokines play a key role in the host inflammatory response to damage by Salmonella typhimurium. It has been revealed that E. faecium increases the expression of proinflammatory and anti-inflammatory cytokines without appearing as a pathogen. Furthermore, E. hirae exerts the gut epithelial barrier function by inducing Th17 [30].

Saccharomyces is well-known nonpathogenic selective probiotic yeast that has been used commercially in the production of probiotic foods. Over the past few decades, $S$. cerevisiae and $S$. boulardii have demonstrated extensive promise as a probiotic treatment [28]. Several studies have demonstrated that $S$. cerevisiae and $S$. boulardii were associated with an increased proportion of Bacteroidetes in the gut microbiota composition and decreased the relative abundance of Firmicutes and Proteobacteria. In addition, this yeast has ability to prevent inflammation by promoting proinflammatory immune function and increasing the production of short-chain fatty acids $[28,29,75,76]$.

\section{Conclusions}

Probiotic bacteria species form a reproducible gut microbiota population in various host bodies and diseases. Various probiotic species have been reported to prevent many degenerative diseases, including obesity, diabetes, cancer, cardiovascular disease, malignancy, liver diseases, and IBD. An imbalance of the gut microbiota composition can lead to several diseases. Probiotics have been proved to modulate gut microbiota composition imbalance by increasing bacteria population, gut epithelium barrier function, and cytokine production. Meanwhile, diets and different nutrients have been reported to productively and markedly shape gut microbiota communities [77-83], further studies should be performed to elucidate the metagenomics relationship between alteration of the gut microbiota composition and probiotic species under different diets or nutrients. A well-designed and appropriate experimental model (in vivo, in vitro, or ex vivo) is suggested to provide insights into the gut microbiota composition and potential commensals for host health. Furthermore, the identification of new probiotics and isolation from microbiome and mixture of probiotic species would be a key pathway for future studies to promote host health.

\section{Abbreviations}

GIT: Gastrointestinal tract

CVD: Cardiovascular disease

LAB: Lactic acid bacteria

HFD: High-fat diet

LPS: Lipopolysaccharide

Treg: T regulatory cell

IL: Interleukin

Th: Thelper cell

DC: Dendritic cell

IBD: Intestinal bowel disease

WSD: Western-style diet

EcN: Escherichia coli Nissle

OMVs: Outer membrane vessels.

\section{Disclosure}

This review article does not contain any studies with human participants or animals performed by any of the authors.

\section{Conflicts of Interest}

The authors declare that there are no potential conflicts of interest regarding the publication of this article.

\section{Acknowledgments}

This research was supported by the National Natural Science Foundation of China (nos. 31772642, 31672457, and 31702125), Hunan Provincial Science and Technology Department (2017NK2322), International Partnership Program of Chinese Academy of Sciences (161643KYSB20160008), and National Key Research and Development Program of China (2016YFD0500504 and 2016YFD0501201). The authors also acknowledge CAS-TWAS President's Fellowship.

\section{References}

[1] G. la Fata, P. Weber, and M. H. Mohajeri, "Probiotics and the Gut Immune System: Indirect Regulation," Probiotics and Antimicrobial Proteins, pp. 1-11, 2017.

[2] A. C. Brown and A. Valiere, "Probiotics and medical nutrition therapy," Nutrition in Clinical Care, vol. 7, no. 2, pp. 56-68, 2004.

[3] E. Metchnikoff, "The prolongation of life: optimistic studies," C. P. Mitchell, Ed., GP Putnam's Sons, New York, NY, USA, 1908.

[4] F. Vergin, "Anti-und Probiotica," Hipokrates, vol. 25, pp. 116-119, 1954.

[5] D. M. Lilly and R. H. Stillwell, "Probiotics: growth-promoting factors produced by microorganisms," Science, vol. 147, no. 3659, pp. 747-748, 1965.

[6] Food and Agriculture Organization (FAO), Guidelines for the Evaluation of Probiotics in Food; Report of a Joint FAO/WHO Working Group on Drafting Guidelines for the Evaluation of Probiotics in Food; FAO: London, ON, Canada, 2002.

[7] C. Hill, F. Guarner, G. Reid et al., "Expert consensus document: the International Scientific Association for Probiotics and Prebiotics consensus statement on the scope and appropriate use of the term probiotic," Nature Reviews Gastroenterology \& Hepatology, vol. 11, no. 8, pp. 506-514, 2014.

[8] P. Markowiak and K. Ślizewska, "Effects of probiotics, prebiotics, and synbiotics on human health," Nutrients, vol. 9, no. 9, article no. 1021, 2017.

[9] M. He and B. Shi, "Gut microbiota as a potential target of metabolic syndrome: The role of probiotics and prebiotics," Cell \& Bioscience, vol. 7, no. 1, article no. 54, 2017.

[10] M.-J. Butel, "Probiotics, gut microbiota and health," Médecine et Maladies Infectieuses, vol. 44, no. 1, pp. 1-8, 2014.

[11] H. Kitazawa, S. Alvarez, A. Suvorov, V. Melnikov, J. Villena, and B. Sánchez, "Recent advances and future perspective in microbiota and probiotics," BioMed Research International, vol. 2015, Article ID 275631, 2015.

[12] S. Lebeer, J. Vanderleyden, and S. C. J. de Keersmaecker, "Host interactions of probiotic bacterial surface molecules: comparison with commensals and pathogens," Nature Reviews Microbiology, vol. 8, no. 3, pp. 171-184, 2010. 
[13] P. A. Bron, P. van Baarlen, and M. Kleerebezem, "Emerging molecular insights into the interaction between probiotics and the host intestinal mucosa," Nature Reviews Microbiology, vol. 10, no. 1, pp. 66-78, 2012.

[14] H. Kitazawa, J. Villena, and A. Susana, Probiotics: Immunobiotics and Immunogenics, CRC Press, 2013.

[15] J. S. Park et al., "Lactobacillus acidophilus improves intestinal inflammation in an acute colitis mouse model by regulation of Th17 and Treg cell balance and fibrosis development," Journal of Medicinal Food, 2018.

[16] K. Khazaie, M. Zadeh, M. W. Khan et al., "Abating colon cancer polyposis by Lactobacillus acidophilus deficient in lipoteichoic acid," Proceedings of the National Acadamy of Sciences of the United States of America, vol. 109, no. 26, pp. 10462-10467, 2012.

[17] L. Chen, Y. Zou, J. Peng et al., "Lactobacillus acidophilus suppresses colitis-associated activation of the IL-23/Th17 axis," Journal of Immunology Research, vol. 2015, Article ID 909514, 2015.

[18] L.-L. Chen, Y.-Y. Zou, F.-G. Lu, F.-J. Li, and G.-H. Lian, "Efficacy profiles for different concentrations of Lactobacillus acidophilus in experimental colitis," World Journal of Gastroenterology, vol. 19, no. 32, pp. 5347-5356, 2013.

[19] M. Lenoir, S. del Carmen, N. G. Cortes-Perez et al., "Lactobacillus casei BL23 regulates Treg and Th17 T-cell populations and reduces DMH-associated colorectal cancer," Journal of Gastroenterology, vol. 51, no. 9, pp. 862-873, 2016.

[20] E. Jacouton, F. Chain, H. Sokol, P. Langella, and L. G. BermúdezHumarán, "Probiotic strain Lactobacillus casei BL23 prevents colitis-associated colorectal cancer," Frontiers in Immunology, vol. 8, article no. 1553, 2017.

[21] H.-S. Lye, T. Kato, W.-Y. Low et al., "Lactobacillus fermentum FTDC 8312 combats hypercholesterolemia via alteration of gut microbiota," Journal of Biotechnology, vol. 262, pp. 75-83, 2017.

[22] N. Nakamoto, T. Amiya, R. Aoki et al., "Commensal Lactobacillus Controls Immune Tolerance during Acute Liver Injury in Mice," Cell Reports, vol. 21, no. 5, pp. 1215-1226, 2017.

[23] J. Zhao et al., "Lactobacillus plantarum CCFM10 alleviating oxidative stress and restoring the gut microbiota in d-galactoseinduced aging mice," Food \& Function, 2018.

[24] L. Xue, J. He, N. Gao et al., "Probiotics may delay the progression of nonalcoholic fatty liver disease by restoring the gut microbiota structure and improving intestinal endotoxemia," Scientific Reports, vol. 7, Article ID 45176, 2017.

[25] R. A. Bagarolli, N. Tobar, A. G. Oliveira et al., "Probiotics modulate gut microbiota and improve insulin sensitivity in DIO mice," The Journal of Nutritional Biochemistry, vol. 50, pp. 1625, 2017.

[26] B. Sánchez, I. González-Rodríguez, S. Arboleya et al., “The effects of bifidobacterium breve on immune mediators and proteome of ht29 cells monolayers," BioMed Research International, vol. 2015, Article ID 479140, 2015.

[27] T. Secher, S. Kassem, M. Benamar et al., "Oral administration of the probiotic strain Escherichia coli Nissle 1917 reduces susceptibility to neuroinflammation and repairs experimental autoimmune encephalomyelitis-induced intestinal barrier dysfunction," Frontiers in Immunology, vol. 8, article no. 1096, 2017.

[28] L. Yu, X.-K. Zhao, M.-L. Cheng et al., "Saccharomyces boulardii Administration Changes Gut Microbiota and Attenuates DGalactosamine-Induced Liver Injury," Scientific Reports, vol. 7, no. 1, article no. 1359, 2017.
[29] A. Everard, S. Matamoros, L. Geurts, N. M. Delzenne, and P. D. Cani, "Saccharomyces boulardii administration changes gut microbiota and reduces hepatic steatosis, low-grade inflammation, and fat mass in obese and type 2 diabetic $\mathrm{db} / \mathrm{db}$ mice," $m B i o$, vol. 5, no. 3, 2014.

[30] R. Daillère, M. Vétizou, N. Waldschmitt et al., "Enterococcus hirae and Barnesiella intestinihominis Facilitate Cyclophosphamide-Induced Therapeutic Immunomodulatory Effects," Immunity, vol. 45, no. 4, pp. 931-943, 2016.

[31] J. Lederberg and A. T. McCray, "Ome sweet omics-a genealogical treasury of words," The Scientist, vol. 15, no. 7, 8 pages, 2001.

[32] R. Sender, S. Fuchs, and R. Milo, "Revised Estimates for the Number of Human and Bacteria Cells in the Body," PLoS Biology, vol. 14, no. 8, Article ID e1002533, 2016.

[33] F. Bäckhed, H. Ding, T. Wang et al., "The gut microbiota as an environmental factor that regulates fat storage," Proceedings of the National Acadamy of Sciences of the United States of America, vol. 101, no. 44, pp. 15718-15723, 2004.

[34] J. G. LeBlanc, C. Milani, G. S. de Giori, F. Sesma, D. van Sinderen, and M. Ventura, "Bacteria as vitamin suppliers to their host: A gut microbiota perspective," Current Opinion in Biotechnology, vol. 24, no. 2, pp. 160-168, 2013.

[35] K. Endt, B. Stecher, S. Chaffron et al., "The microbiota mediates pathogen clearance from the gut lumen after non-typhoidal salmonella diarrhea," PLoS Pathogens, vol. 6, no. 9, Article ID e01097, 2010.

[36] J. L. Round and S. K. Mazmanian, "The gut microbiota shapes intestinal immune responses during health and disease," Nature Reviews Immunology, vol. 9, no. 5, pp. 313-323, 2009.

[37] S. M. Collins, M. Surette, and P. Bercik, "The interplay between the intestinal microbiota and the brain," Nature Reviews Microbiology, vol. 10, no. 11, pp. 735-742, 2012.

[38] P. Bin, S. Liu, S. Chen et al., "The effect of aspartate supplementation on the microbial composition and innate immunity on mice," Amino Acids, vol. 49, no. 12, pp. 2045-2051, 2017.

[39] Q. Feng, S. Liang, H. Jia et al., "Gut microbiome development along the colorectal adenoma-carcinoma sequence," Nature Communications, vol. 6, 2015.

[40] N. S. Barteneva, Y. Baiken, E. Fasler-Kan et al., "Extracellular vesicles in gastrointestinal cancer in conjunction with microbiota: On the border of Kingdoms," Biochimica et Biophysica Acta (BBA) - Reviews on Cancer, vol. 1868, no. 2, pp. 372-393, 2017.

[41] M. J. Saez-Lara, C. Gomez-Llorente, J. Plaza-Diaz, and A. Gil, "The role of probiotic lactic acid bacteria and bifidobacteria in the prevention and treatment of inflammatory bowel disease and other related diseases: a systematic review of randomized human clinical trials," BioMed Research International, vol. 2015, Article ID 505878, 15 pages, 2015.

[42] V. Nobili, L. Putignani, A. Mosca et al., "Bifidobacteria and lactobacilli in the gut microbiome of children with non-alcoholic fatty liver disease: which strains act as health players?" Archives of Medical Science, vol. 14, no. 1, pp. 81-87, 2018.

[43] F. Li, K. Duan, C. Wang, C. McClain, and W. Feng, "Probiotics and alcoholic liver disease: treatment and potential mechanisms," Gastroenterology Research and Practice, vol. 2016, pp. 111, 2016.

[44] M. J. Claesson, I. B. Jeffery, S. Conde et al., "Gut microbiota composition correlates with diet and health in the elderly," Nature, vol. 488, no. 7410, pp. 178-184, 2012. 
[45] Y.-J. Zhang, S. Li, R.-Y. Gan, T. Zhou, D.-P. Xu, and H.-B. Li, "Impacts of gut bacteria on human health and diseases," International Journal of Molecular Sciences, vol. 16, no. 4, pp. 74937519, 2015.

[46] W. H. W. Tang and S. L. Hazen, "The contributory role of gut microbiota in cardiovascular disease," The Journal of Clinical Investigation, vol. 124, no. 10, pp. 4204-4211, 2014.

[47] F. Z. Marques, E. Nelson, P.-Y. Chu et al., "High Fibre Diet and Acetate Supplementation Change the Gut Microbiota and Prevent the Development of Hypertension and Heart Failure in DOCA-Salt Hypertensive Mice," Circulation, 2017.

[48] J. A. Foster and K.-A. McVey Neufeld, "Gut-brain axis: How the microbiome influences anxiety and depression," Trends in Neurosciences, vol. 36, no. 5, pp. 305-312, 2013.

[49] Y. Liang, S. Liang, Y. Zhang et al., "Oral Administration of compound probiotics ameliorates HFD-induced gut microbe dysbiosis and chronic metabolic inflammation via the G proteincoupled receptor 43 in non-alcoholic fatty liver disease rats," Probiotics and Antimicrobial Proteins, 2018.

[50] R. Gu, Z. Yang, X. Chen et al., "The effect of Lactobacillus rhamnosus hsryfm 1301 on the intestinal microbiota of a hyperlipidemic rat model," BMC Complementary and Alternative Medicine, vol. 14, p. 386, 2014.

[51] R. V. Bubnov, L. P. Babenko, L. M. Lazarenko et al., "Comparative study of probiotic effects of Lactobacillus and Bifidobacteria strains on cholesterol levels, liver morphology and the gut microbiota in obese mice," EPMA Journal, vol. 8, no. 4, pp. 357376, 2017.

[52] J. Karczewski, F. J. Troost, I. Konings et al., "Regulation of human epithelial tight junction proteins by Lactobacillus plantarum in vivo and protective effects on the epithelial barrier," American Journal of Physiology-Gastrointestinal and Liver Physiology, vol. 298, no. 6, pp. G851-G859, 2010.

[53] F. A. Carvalho, J. D. Aitken, M. Vijay-Kumar, and A. T. Gewirtz, "Toll-like receptor-gut microbiota interactions: Perturb at your own risk!," Annual Review of Physiology, vol. 74, pp. 177-198, 2012.

[54] Z. J. Liu, P. K. Yadav, J. L. Su, J. S. Wang, and K. Fei, "Potential role of Th17 cells in the pathogenesis of in flammatory bowel disease," World Journal of Gastroenterology, vol. 15, no. 46, pp. 5784-5788, 2009.

[55] Y. He, M. Gao, Y. Cao, H. Tang, S. Liu, and Y. Tao, "Nuclear localization of metabolic enzymes in immunity and metastasis," Biochimica et Biophysica Acta (BBA) - Reviews on Cancer, vol. 1868, no. 2, pp. 359-371, 2017.

[56] W. Ren, S. Chen, L. Zhang et al., "Interferon tau affects mouse intestinal microbiota and expression of IL-17," Mediators of Inflammation, vol. 2016, Article ID 2839232, 9 pages, 2016.

[57] F. Aragón, S. Carino, G. Perdigón, and A. De Moreno de LeBlanc, "The administration of milk fermented by the probiotic Lactobacillus casei CRL 431 exerts an immunomodulatory effect against a breast tumour in a mouse model," Immunobiology, vol. 219, no. 6, pp. 457-464, 2014.

[58] J. Hu, C. Wang, L. Ye et al., "Anti-tumour immune effect of oral administration of Lactobacillus plantarum to CT26 tumourbearing mice," Journal of Biosciences, vol. 40, no. 2, pp. 269-279, 2015.

[59] I. Trabelsi, N. Ktari, S. Ben Slima, K. Hamden, and R. Ben Salah, "Effect of a probiotic Lactobacillus plantarum TN8 strain on trinitrobenzene sulphonic acid-induced colitis in rats," Journal of Animal Physiology and Animal Nutrition, vol. 101, no. 2, pp. 311-319, 2017.
[60] R. Ben Salah, I. Trabelsi, R. Ben Mansour, S. Lassoued, H. Chouayekh, and S. Bejar, "A new Lactobacillus plantarum strain, TN8, from the gastro intestinal tract of poultry induces high cytokine production," Anaerobe, vol. 18, no. 4, pp. 436-444, 2012.

[61] E. K. Persson, C. L. Scott, A. M. Mowat, and W. W. Agace, "Dendritic cell subsets in the intestinal lamina propria: ontogeny and function," European Journal of Immunology, vol. 43, no. 12, pp. 3098-3107, 2013.

[62] L. Fu, J. Song, C. Wang, S. Fu, and Y. Wang, "Bifidobacterium infantis potentially alleviates shrimp tropomyosin-induced allergy by tolerogenic dendritic cell-dependent induction of regulatory $\mathrm{T}$ cells and alterations in gut microbiota," Frontiers in Immunology, vol. 8, article no. 1536, 2017.

[63] D. Srutkova, M. Schwarzer, T. Hudcovic et al., "Bifidobacterium longum CCM 7952 promotes epithelial barrier function and prevents acute dss-induced colitis in strictly strain-Specific manner," PLoS ONE, vol. 10, no. 7, Article ID e0134050, 2015.

[64] F. Turroni, E. Özcan, C. Milani et al., "Glycan cross-feeding activities between bifidobacteria under in vitro conditions," Frontiers in Microbiology, vol. 6, Article ID 01030, 2015.

[65] B. O. Schroeder, G. M. H. Birchenough, M. Ståhlman et al., "Bifidobacteria or Fiber Protects against Diet-Induced MicrobiotaMediated Colonic Mucus Deterioration," Cell Host \& Microbe, 2017.

[66] H. M. Savignac, M. Tramullas, B. Kiely, T. G. Dinan, and J. F. Cryan, "Bifidobacteria modulate cognitive processes in an anxious mouse strain," Behavioural Brain Research, vol. 287, pp. 5972, 2015.

[67] J. Arseneault-Bréard, I. Rondeau, K. Gilbert et al., "Combination of Lactobacillus helveticus R0052 and Bifidobacterium longum R0175 reduces post-myocardial infarction depression symptoms and restores intestinal permeability in a rat model," British Journal of Nutrition, vol. 107, no. 12, pp. 1793-1799, 2012.

[68] M. Schlee, J. Wehkamp, A. Altenhoefer, T. A. Oelschlaeger, E. F. Stange, and K. Fellermann, "Induction of human $\beta$-defensin 2 by the probiotic Escherichia coli Nissle 1917 is mediated through flagellin," Infection and Immunity, vol. 75, no. 5, pp. 2399-2407, 2007.

[69] G. Liu, W. Ren, J. Fang, and C. A. Hu, "L-Glutamine and L-arginine protect against enterotoxigenic Escherichia coli infection via intestinal innate immunity in mice," Amino Acids, pp. 1-10, 2017.

[70] A. A. Zyrek, C. Cichon, S. Helms, C. Enders, U. Sonnenborn, and M. A. Schmidt, "Molecular mechanisms underlying the probiotic effects of Escherichia coli Nissle 1917 involve ZO-2 and PKCzeta redistribution resulting in tight junction and epithelial barrier repair," Cellular Microbiology, vol. 9, no. 3, pp. 804-816, 2007.

[71] M.-J. Fábrega, A. Rodríguez-Nogales, J. Garrido-Mesa et al., "Intestinal anti-inflammatory effects of outer membrane vesicles from Escherichia coli Nissle 1917 in DSS-experimental colitis in mice," Frontiers in Microbiology, vol. 8, article no. 1274, 2017.

[72] M.-A. Cañas, R. Giménez, M.-J. Fábrega, L. Toloza, L. Baldomà, and J. Badia, "Outer membrane vesicles from the probiotic Escherichia coli Nissle 1917 and the commensal ECOR12 enter intestinal epithelial cells via clathrin-dependent endocytosis and elicit differential effects on DNA damage," PLoS ONE, vol. 11, no. 8, Article ID e0160374, 2016.

[73] E. Tarasova, E. Yermolenko, V. Donets et al., "The influence of probiotic Enterococcus faecium strain L5 on the microbiota 
and cytokines expression in rats with dysbiosis induced by antibiotics," Beneficial Microbes, vol. 1, no. 3, pp. 265-270, 2010.

[74] A. Fusco, V. Savio, M. Cammarota, A. Alfano, C. Schiraldi, and G. Donnarumma, "Beta-Defensin-2 and Beta-Defensin-3 reduce intestinal damage caused by Salmonella typhimurium modulating the expression of cytokines and enhancing the probiotic activity of enterococcus faecium," Journal of Immunology Research, vol. 2017, 9 pages, 2017.

[75] M. Li, L. Zhu, A. Xie, and J. Yuan, "Oral Administration of Saccharomyces boulardii Ameliorates Carbon Tetrachloride-Induced Liver Fibrosis in Rats via Reducing Intestinal Permeability and Modulating Gut Microbial Composition," Inflammation, vol. 38, no. 1, pp. 170-179, 2014.

[76] P. Brun et al., "Saccharomyces boulardii CNCM I-745 supplementation reduces gastrointestinal dysfunction in an animal model of IBS," Saccharomyces boulardii CNCM I-745 supplementation reduces gastrointestinal dysfunction in an animal model of IBS, vol. 12, no. 7, Article ID e0181863, 2017.

[77] G. S. Kopeina, V. V. Senichkin, and B. Zhivotovsky, "Caloric restriction - A promising anti-cancer approach: From molecular mechanisms to clinical trials," Biochimica et Biophysica Acta (BBA) - Reviews on Cancer, vol. 1867, no. 1, pp. 29-41, 2017.

[78] A. Morandi and S. Indraccolo, "Linking metabolic reprogramming to therapy resistance in cancer," Biochimica et Biophysica Acta (BBA) - Reviews on Cancer, vol. 1868, no. 1, pp. 1-6, 2017.

[79] W. Ren, P. Wang, J. Yan et al., "Melatonin alleviates weanling stress in mice: Involvement of intestinal microbiota," Journal of Pineal Research, vol. 2, 2018.

[80] J. Yin, W. Ren, X. Huang, T. Li, and Y. Yin, "Protein restriction and cancer," Biochimica et Biophysica Acta (BBA) - Reviews on Cancer, vol. 1869, no. 2, pp. 256-262, 2018.

[81] J. Costa, "Glycoconjugates from extracellular vesicles: Structures, functions and emerging potential as cancer biomarkers," Biochimica et Biophysica Acta (BBA) - Reviews on Cancer, vol. 1868, no. 1, pp. 157-166, 2017.

[82] G. Liu, S. Chen, G. Guan et al., "Chitosan Modulates Inflammatory Responses in Rats Infected with Enterotoxigenic Escherichia coli," Mediators of Inflammation, vol. 2016, Article ID 7432845, 6 pages, 2016.

[83] J. Yin, H. Han, Y. Li et al., "Lysine Restriction Affects Feed Intake and Amino Acid Metabolism via Gut Microbiome in Piglets," Cellular Physiology and Biochemistry, pp. 1749-1761, 2017. 


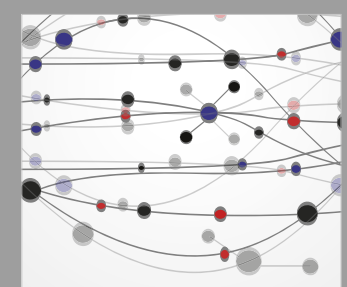

The Scientific World Journal
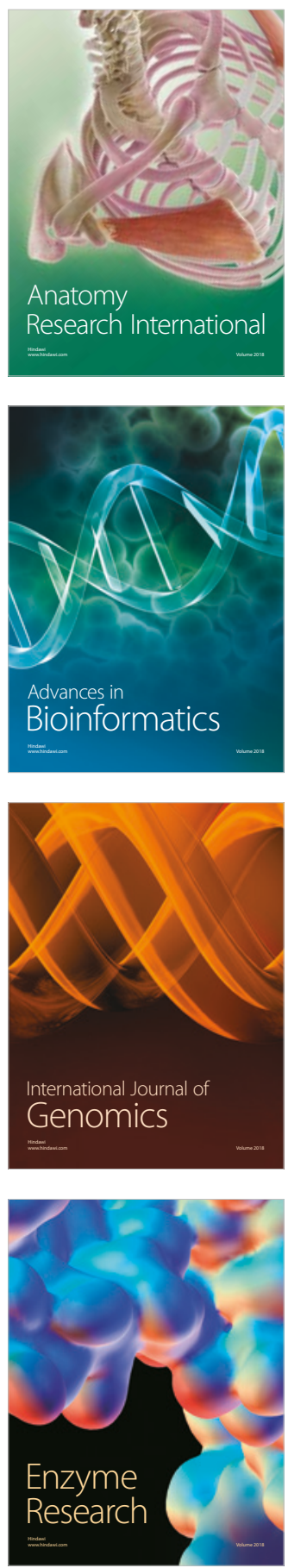
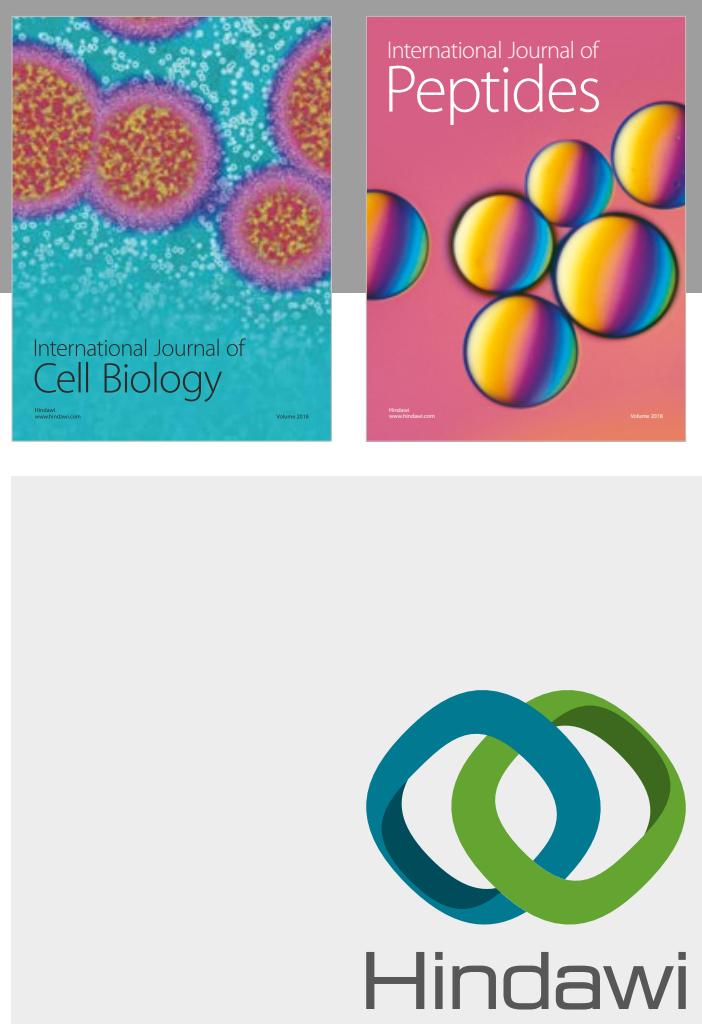

Submit your manuscripts at

www.hindawi.com
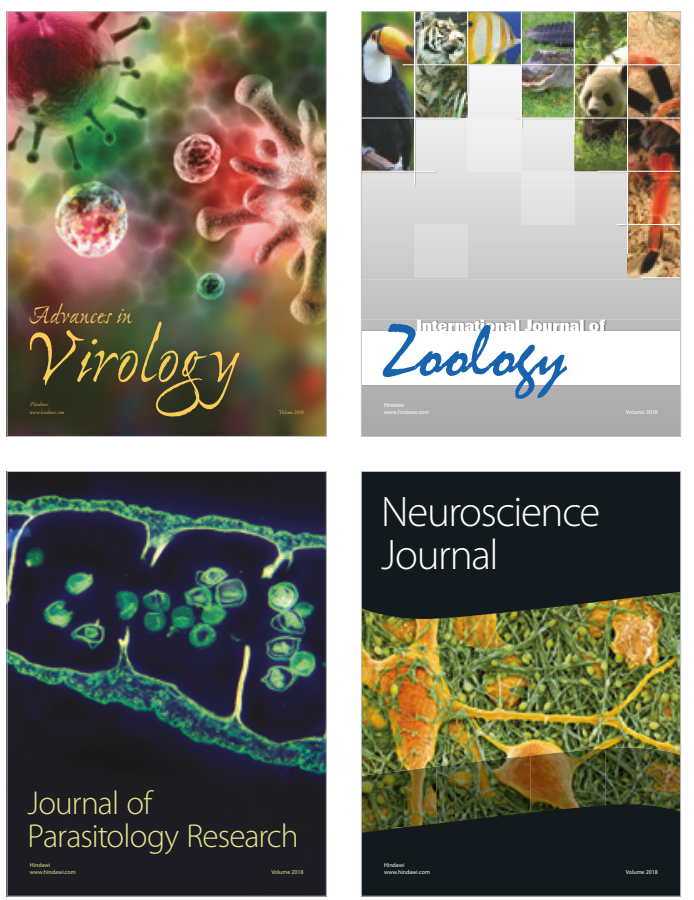
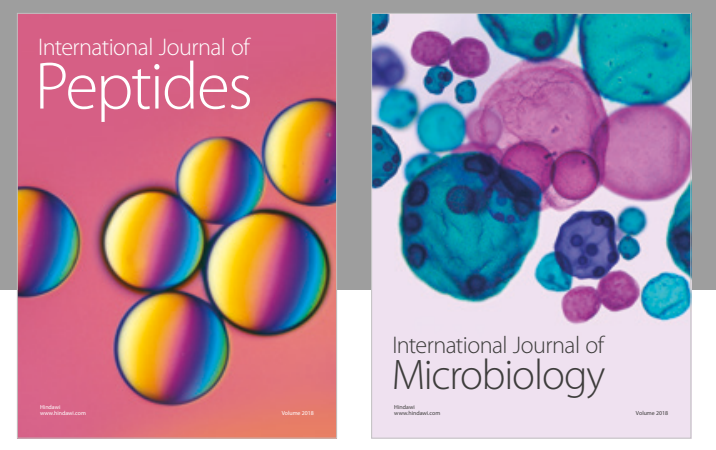

nternational Journal of Microbiology
Journal of
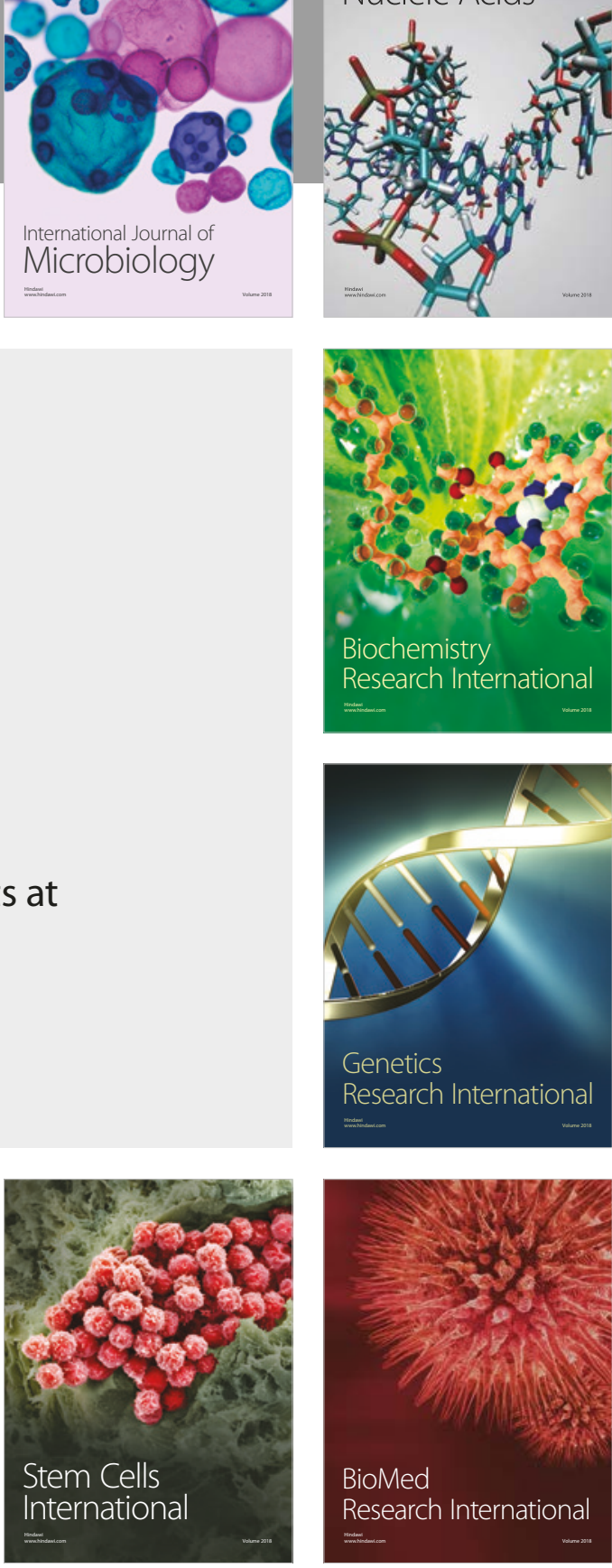
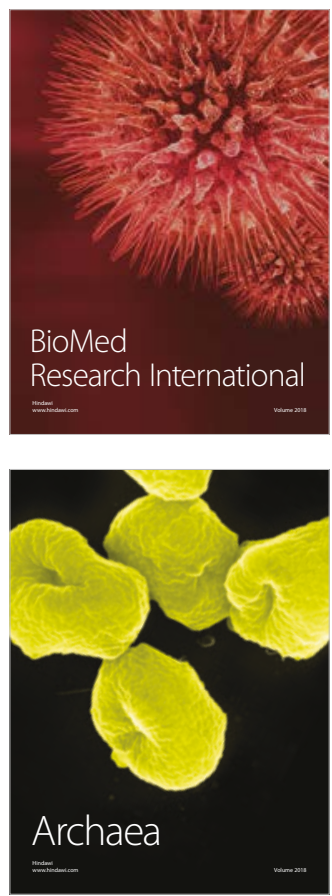